\title{
Chiral Discrimination of Tryptophan Enantiomers via (1R, 2R)-2-Amino-1, 2-Diphenyl Ethanol Modified Interface
}

\author{
Juan Zhou, Qiao Chen, Li-lan Wang, Yong-hua Wang, and Ying-zi Fu \\ Key Laboratory on Luminescence and Real-Time Analysis of Ministry of Education, College of Chemistry and Chemical Engineering, \\ Southwest University, Chongqing 400715, China \\ Correspondence should be addressed to Ying-zi Fu, fyzc@swu.edu.cn
}

Received 5 May 2011; Accepted 18 June 2011

Academic Editor: Valentin Mirceski

Copyright ( $\odot 2011$ Juan Zhou et al. This is an open access article distributed under the Creative Commons Attribution License, which permits unrestricted use, distribution, and reproduction in any medium, provided the original work is properly cited.

The paper reported that a simple chiral selective interface constructed by (1R, 2R)-2-amino-1, 2-diphenyl ethanol had been developed to discriminate tryptophan enantiomers. Cyclic voltammetry (CV) and electrochemical impedance spectroscopy (EIS) were used for the characteristic analysis of the electrode. The results indicated that the interface showed stable and sensitive property to determine the tryptophan enantiomers. Moreover, it exhibited the better stereoselectivity for L-tryptophan than that for D-tryptophan. The discrimination characteristics of the chiral selective interface for discriminating tryptophan enantiomers, including the response time, the effect of tryptophan enantiomers concentration, and the stability, were investigated in detail. In addition, the chiral selective interface was used to determine the enantiomeric composition of L- and D-tryptophan enantiomer mixtures by measuring the relative change of the peak current as well as in pure enantiomeric solutions. These results suggested that the chiral selective interface has the potential for enantiomeric discrimination of tryptophan enantiomers.

\section{Introduction}

Chirality is an intrinsic property of most biological molecules. Chiral molecules may create a totally different effect on many biological processes in nature and life [1]. One enantiomer of drug may be more active or toxic than another isomer [2]. Due to the dramatic and total different effect of enantiomers compounds on human beings, chiral recognition is becoming a very active area in food, environment, clinic, medical, and pharmaceutical industry. Various techniques have been applied to discriminate and separate the racemic compounds [3-11]. Among these methods, electrochemistry with high sensitivity, simpleness, low cost, low-power requirements, and high compatibility has been a preferable approach for chiral recognition.

One of the principal goals for chiral recognition is to construct an effectual chiral selective system, which should have recognition site for certain chiral enantiomers [12]. As one of amino alcohol derivatives, 2-amino-1, 2-diphenyl ethanol (ADE) is a chiral reagent having two asymmetric carbons in their structure. The amino group may recruit a negatively charged side chain and the hydroxy group may recruit hydro- gen bonding donor/acceptor system. ADE enantiomers have been applied to prepare the chiral intermediates of bioactive molecule and have been used as a chiral ligand to induce asymmetric transformation $[13,14]$. And it was a stuff to prepare chiral selector showing excellent enantioseparation ability [15].

Tryptophan (Trp) has a great influence on metabolic pathways [16]. L-tryptophan (L-Trp) is an essential amino acid in proteins, food, pharmaceuticals, and the unbalance or deficiency of L-Trp may cause several chronic diseases $[17,18]$. As a nonprotein amino acid, D-tryptophan (DTrp) does not join in metabolism pathways, but it has special physiological properties in food, feed, and medical fields. And D-Trp is an important intermediate to prepare synthetic peptide antibiotics and immunosuppressive agents in the pharmaceutical industry [19]. Thus, numerous efforts have been made to find an applicable method for the chiral analysis of Trp enantiomers in pharmacy [20,21].

In this paper, $(1 \mathrm{R}, 2 \mathrm{R})$-ADE which has two chiral centers was utilized as a selective agent to discriminate Trp enantiomers. It was covalently modified on the gold electrodes to construct the chiral selective interface. Cyclic voltammetry 
(CV) and electrochemical impedance spectroscopy (EIS) were used to analyze the characteristics of the interface. The experimental results indicated that the resulting electrodes could be used to discriminate Trp enantiomers, and the chiral selective interface might have good prospects for quantitative analyzing enantiomer composition in the pharmaceutical samples.

\section{Experimental}

2.1. Materials and Reagents. (1R, 2R)-2-amino-1,2-diphenyl ethanol ((1R, 2R)-ADE) and 3-mercaptopropionic acid (MPA) were obtained from Koch-Light Laboratories Ltd (Coinbrook, Bucks., UK). N-(3-dimethylaminopropyl)-Nethylcarbodiimidehydrochloride (EDC), N-hydroxy succinimide (NHS), and tryptophan (Trp) enantiomers were purchased from Sigma Chemical Co (St. Louis, MO, USA). Phosphate buffer saline was prepared with $\mathrm{KH}_{2} \mathrm{PO}_{4}$ and $\mathrm{Na}_{2} \mathrm{HPO}_{4}$ which contained $0.1 \mathrm{M} \mathrm{KCl}$ (PBS, pH 6.75). All other reagents were commercially available and of analytical grade. Double distilled water was used throughout the experiments.

2.2. Apparatus. CV and EIS detections were performed with a CHI 660D electrochemistry workstation (Shanghai Chenhua Instruments Co., China). A conventional threeelectrode system was employed with bare gold or the modified electrodes ( $\mathrm{Au}, \Phi=4 \mathrm{~mm}$ ) as the working electrodes, a platinum wire auxiliary electrode as the counter electrode, and a saturated calomel reference electrode (SCE) as the reference electrode. $\mathrm{CV}$ and EIS were measured in $5 \mathrm{mM}$ $\left[\mathrm{Fe}(\mathrm{CN})_{6}\right]^{4-/ 3-}$ solution (0.1 M PBS, pH 6.75).

2.3. The Construction of the Chiral Selective Interface. Gold electrodes were firstly polished with 1.0, 0.3, $0.05 \mu \mathrm{m}$ alumina slurries and ultrasonicated in double distilled water, ethanol, and double distilled water for $5 \mathrm{~min}$, respectively. A self-assembled MPA monolayer formed on the bare gold electrode surface through Au-S bond in the $5 \mathrm{mM}$ MPA ethanol solution for $12 \mathrm{~h}$ at $4^{\circ} \mathrm{C}$. Then, the carboxyl groups of immobilized MPA were activated by EDC and NHS for $1.5 \mathrm{~h}$ at room temperature. Following that, the electrode was immersed in $5 \mathrm{mM}(1 \mathrm{R}, 2 \mathrm{R})-\mathrm{ADE}$ ethanol solution overnight at room temperature to form the amide through the reaction of carboxyl and amino group. Thus, a chiral selective interface with (1R, 2R)-ADE had been constructed. The construction process of the interface was schematically shown in Figure 1.

\section{Results and Discussion}

3.1. The Electrochemical Characteristics of the Chiral Selective Interface. Electrochemical approaches are powerful tools to study the interface properties of modified electrodes. The cyclic voltammograms (CVs) of different modified electrodes in $5 \mathrm{mM}\left[\mathrm{Fe}(\mathrm{CN})_{6}\right]^{4-/ 3-}$ solution at a scan rate of $100 \mathrm{mV} \cdot \mathrm{s}^{-1}$ were shown in Figure 2(a). Clear redox peak was shown at the bare gold electrode (curve a). After gold

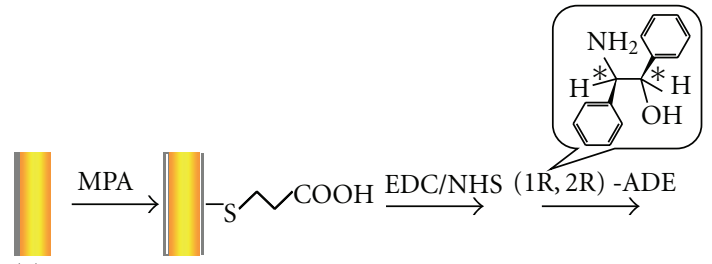

(a)

(b)

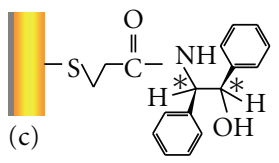

FIGURE 1: Schematic illustration of the modified electrode: (a) Bare Au electrode; (b) MPA self-assembled Au electrode; (c) (1R, 2R)ADE-MPA modified Au electrode.

electrode was dipped into MPA, the redox peak current had diminished (curve b), indicating the self-assembled MPA monolayer had partly blocked the electron-transfer of $\left[\mathrm{Fe}(\mathrm{CN})_{6}\right]^{4-/ 3-}$ on the gold electrode surface. While the electrode had been activated by EDC/NHS and reacted with $(1 \mathrm{R}, 2 \mathrm{R})-\mathrm{ADE}$, the redox peak current had increased (curve c), revealing that the $(1 \mathrm{R}, 2 \mathrm{R})$-ADE could provide the conducting bridge for the electron transfer of $\left[\mathrm{Fe}(\mathrm{CN})_{6}\right]^{4-/ 3-}$.

As shown in Figure 2(b), the EIS diagram introduced the construction processes of the modified electrodes. Bare gold electrode had a small semicircle (curve a), while the MPA monolayer exhibited a remarkable semicircle (curve b). After the electrode was modified with $(1 \mathrm{R}, 2 \mathrm{R})$-ADE, the $R_{\mathrm{et}}$ decreased obviously (curve $\mathrm{c}$ ). This confirmed that the chiral interface was assembled successfully.

The CVs of the resulting electrodes in $\left[\mathrm{Fe}(\mathrm{CN})_{6}\right]^{4-/ 3-}$ solution at different scan rates were investigated too. From Figure 3, the potential and peak current depended on the scan rate, and both the anodic and cathodic peak currents were proportional to the square root of scan rates in the range of $50-450 \mathrm{mV} \cdot \mathrm{s}^{-1}$ (inset of Figure 3 ), suggesting a diffusion controlled redox process $[22,23]$. Furthermore, while the modified electrodes were scanned 50 cycles in $\left[\mathrm{Fe}(\mathrm{CN})_{6}\right]^{4-/ 3-}$ at $100 \mathrm{mV} \cdot \mathrm{s}^{-1}$, the relative standard deviation (RSD) of peak currents was $0.8 \%(n=6)$, indicating a permissible stability of this interface.

3.2. Enantioselective Responses of Trp Enantiomers on the $(1 R, 2 R)-A D E$ Interface. After the modified electrodes were immersed in $5 \mathrm{mM} \mathrm{L}$ - and D-Trp solutions for $90 \mathrm{~min}$, the redox peak currents were decreased (Figure 4(a)), and obvious difference of current between L- and D-Trp was observed (curve $\mathrm{c}$ and $\mathrm{d}$ ). $I_{\mathrm{L}}$ (the peak current of L-Trp) was lower than $I_{\mathrm{D}}$ (the peak current of D-Trp), and $\Delta I\left(\Delta I=I_{\mathrm{D}}-I_{\mathrm{L}}\right)$ was $11.8 \mu \mathrm{A}$, hinting the interaction effect between $(1 \mathrm{R}, 2 \mathrm{R})$ ADE and L-Trp was stronger than that of D-Trp. Figure 4(b) illustrated the electron-transfer resistance of the modified electrodes before and after immersed in $5 \mathrm{mM} \mathrm{L-}$ and D-Trp solutions for $90 \mathrm{~min}$. At the beginning, the two electrodes had the similar $R_{\text {et }}$ (curve a and b), after L- and D-Trp were adsorbed on the electrode surface, the difference of $R_{\mathrm{et}}$ 


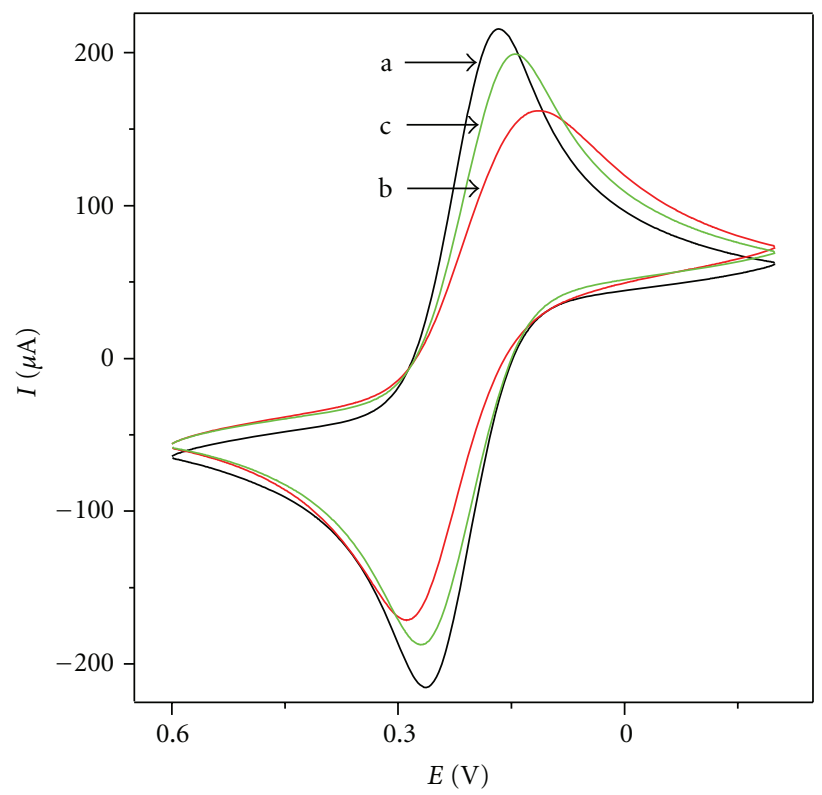

(A)

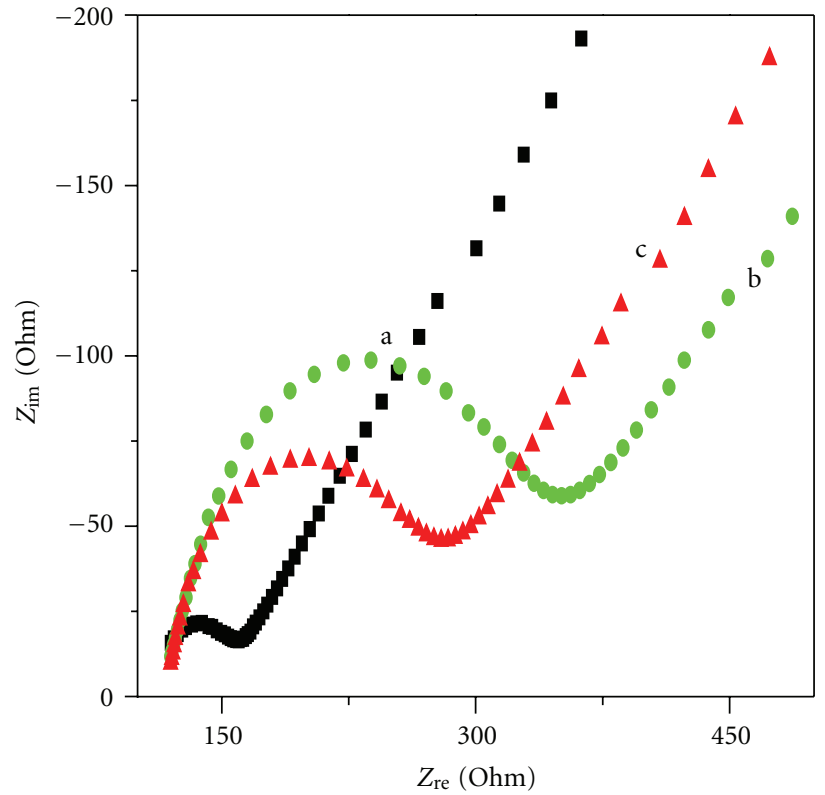

(B)

Figure 2: Cyclic voltammograms (A) and electrochemical impedance spectroscopy (B) recorded of the modified electrodes; (a) Bare $\mathrm{Au}$ electrode; (b) MPA self-assembled Au electrode; (c) (1R, 2R)-ADE-MPA modified Au electrode. Supporting electrolyte, 5 mM $\left[\mathrm{Fe}(\mathrm{CN})_{6}\right]^{4-/ 3-}(0.1 \mathrm{M}$ PBS, $\mathrm{pH} 6.75), 100 \mathrm{mV} \cdot \mathrm{s}^{-1}$.

between L-Trp $\left(R_{\mathrm{et}}=440.8 \Omega\right)$ and D-Trp $\left(R_{\mathrm{et}}=414.4 \Omega\right)$ was enlarged (curve $\mathrm{c}$ and $\mathrm{d}$ ). This result was consistent with the $\mathrm{CV}$.

According to the above results, the stereoselective interaction was exhibited between the modified electrodes and Lor D-Trp, that is to say, the $(1 \mathrm{R}, 2 \mathrm{R})-\mathrm{ADE}$ interface could chiral recognize Trp enantiomers, and the recognition degree for L-Trp is larger than that of D-Trp. The phenomenon probably results in different reacted kinetic or thermodynamic mechanisms between $(1 \mathrm{R}, 2 \mathrm{R})$-ADE interface and Trp enantiomers. First, the hydroxy group of the $(1 \mathrm{R}, 2 \mathrm{R})$ ADE may recruit hydrogen bonding donor/acceptor system $[13,14]$, it may interact with Trp enantiomers to form hydrogen-bond in different mode, leading the different chiral matches. Secondly, because the stereospecific blockade of phenyl group in $(1 \mathrm{R}, 2 \mathrm{R})-\mathrm{ADE}$, the $\mathrm{NH}_{2}$ group is repelled D-Trp stronger than L-Trp, leading the arrangements of L-Trp favourable. In addition, the different van-der-Waals' interactions between the $\operatorname{Trp}$ enantiomers and $(1 \mathrm{R}, 2 \mathrm{R})$ $\mathrm{ADE}$ may be responsible to the chiral recognition [24-26]. In other words, the $(1 \mathrm{R}, 2 \mathrm{R})$-ADE interface is matched to L-Trp better. This should provide useful information for discriminating Trp enantiomers in pharmaceuticals.

\subsection{The Discrimination Characteristics of the Chiral Selective} Interface. The reaction time of the modified electrodes was studied in $5 \mathrm{mM} \mathrm{L}$ - or D-Trp solutions in the range of 10 to $100 \mathrm{~min}$. From the data of Table 1, both the peak currents of L- and D-Trp decreased with increasing of the immersed time. In addition, the relative current change of L-Trp was always much higher than that of D-Trp. While the immersed

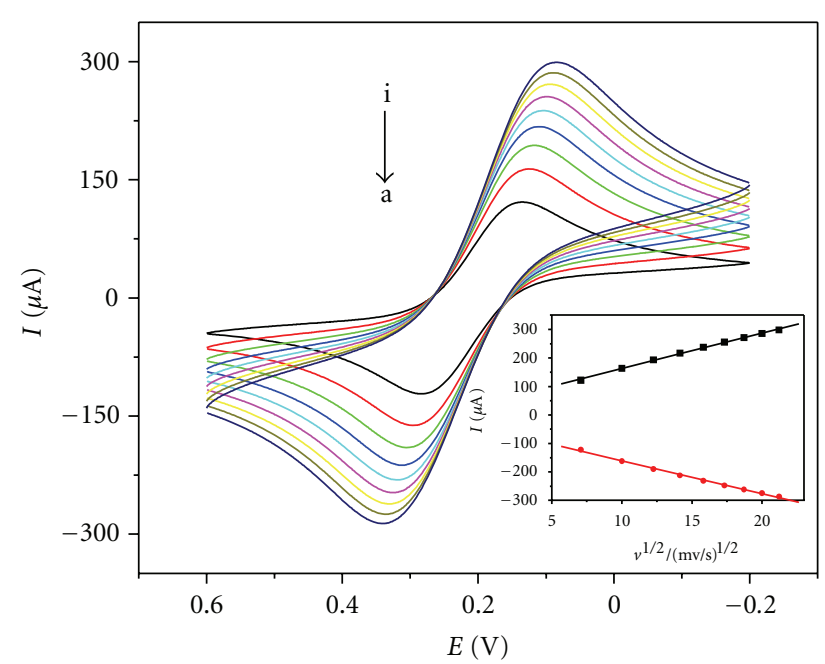

Figure 3: Cyclic voltammograms of the modified electrode at different scan rates (from a to i): 50, 100, 150, 200, 250, 300, 350,400 , and $450 \mathrm{mV} \cdot \mathrm{s}^{-1}$ in $5 \mathrm{mM}\left[\mathrm{Fe}(\mathrm{CN})_{6}\right]^{4-/ 3-}$ solution. All potentials are given versus SCE. The inset shows the dependence of redox peak currents on the square root of scan rates.

time was up to $90 \mathrm{~min}$, the currents had no further change, disclosed Trp enantiomers had been saturated adsorption at the modified electrode. Therefore, $90 \mathrm{~min}$ was used as immersed time of all subsequent assays.

The modified electrodes were used to react with Land D-Trp in the concentration range of 1 to $5 \mathrm{mM}$. From Figure 5, linear relationship was observed between 


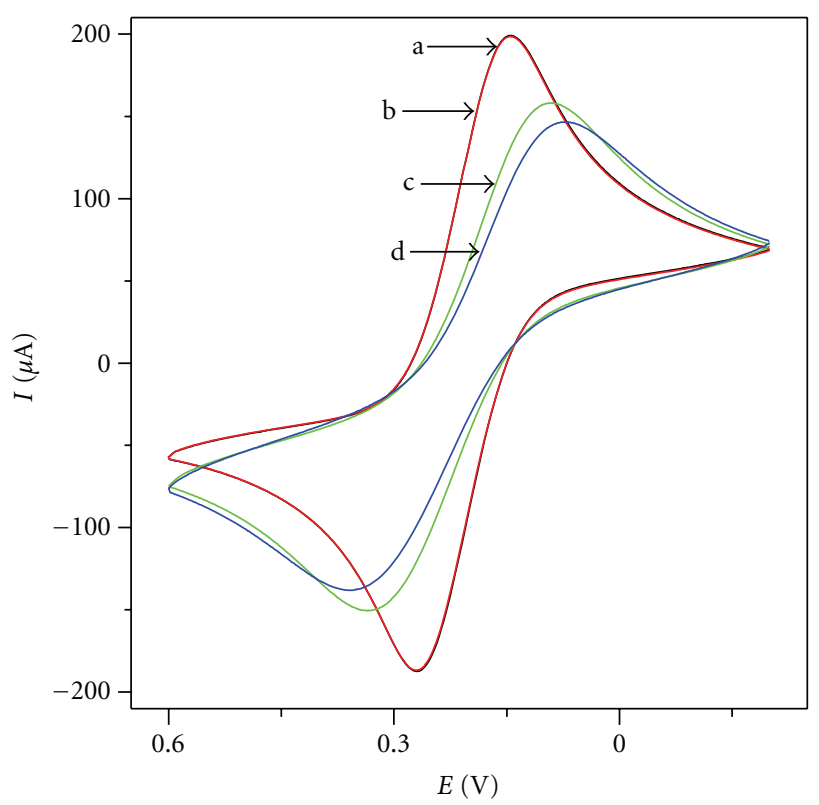

(A)

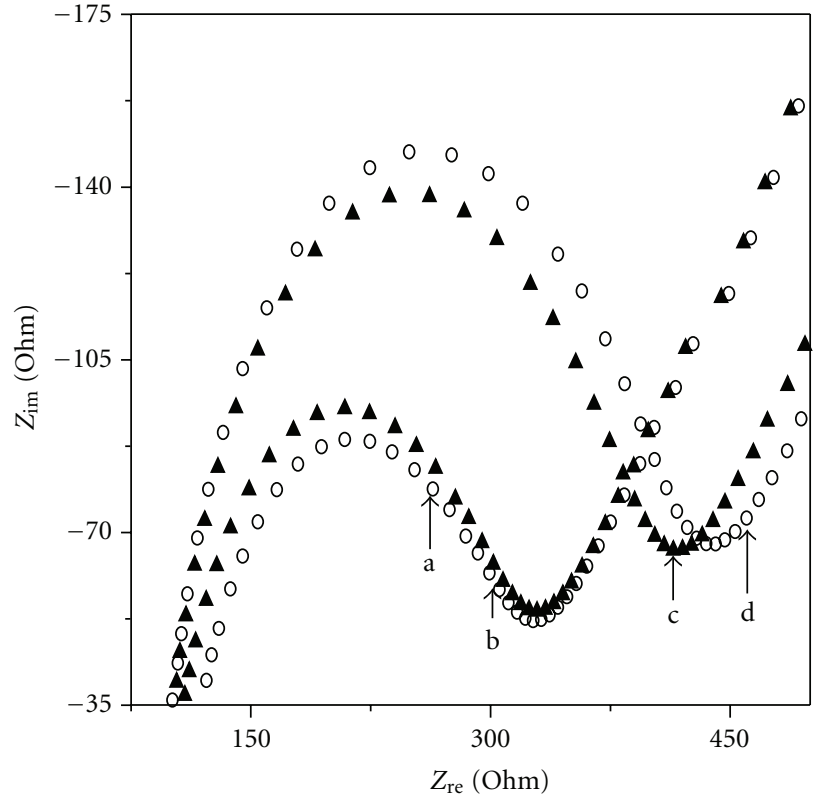

(B)

Figure 4: Cyclic voltammograms (A) and electrochemical impedance spectroscopy (B) of enantioselective responses: (a) and (b) (1R, 2R)ADE-MPA modified Au electrode. (c) 5 mM D-Trp reacted with the modified electrode. (d) 5 mM L-Trp reacted with the modified electrode.

TABLE 1: The current peaks response of chiral interface exposed to L- and D-Trp for different time periods.

\begin{tabular}{lccc}
\hline \multirow{2}{*}{ Immersed time/(min) } & \multicolumn{3}{c}{ Current response } \\
& $I_{\mathrm{D}} / \mu \mathrm{A}$ & $I_{\mathrm{L}} / \mu \mathrm{A}$ & $\Delta I=I_{\mathrm{D}}-I_{\mathrm{L}}(\mu \mathrm{A})$ \\
\hline 0 & 196.2 & 197.6 & -1.4 \\
10 & 193.4 & 192.2 & 1.2 \\
20 & 192.1 & 187.9 & 4.2 \\
30 & 189.3 & 183.8 & 5.5 \\
40 & 185.2 & 178.9 & 6.3 \\
50 & 179.6 & 172 & 7.6 \\
60 & 174.1 & 164 & 10.1 \\
70 & 170.6 & 161.1 & 9.5 \\
80 & 163.6 & 154.2 & 9.4 \\
90 & 158.1 & 146.3 & 11.8 \\
100 & 156 & 145.8 & 10.2 \\
\hline
\end{tabular}

the relative change of oxidation peak currents and the concentration of Trp enantiomers. In contrast with D-Trp, the recognition of L-Trp still kept obvious with increasing of Trp enantiomers concentration.

The stability of the modified electrodes to discriminate Trp enantiomers had been investigated. The modified electrodes were divided into two groups and immersed, respectively, in $5 \mathrm{mM}$ Trp enantiomers solutions for $90 \mathrm{~min}$ at room temperature. After interacting with D-Trp, the modified electrode was scanned 50 circles by $\mathrm{CV}$ at $100 \mathrm{mV} \cdot \mathrm{s}^{-1}$. The relative standard deviation (RSD) of peak currents was $0.57 \%(n=6)$, and the case of L-Trp was $0.81 \%(n=6)$. These demonstrated that the resulting electrodes had good stability to discriminate L- and D-Trp.

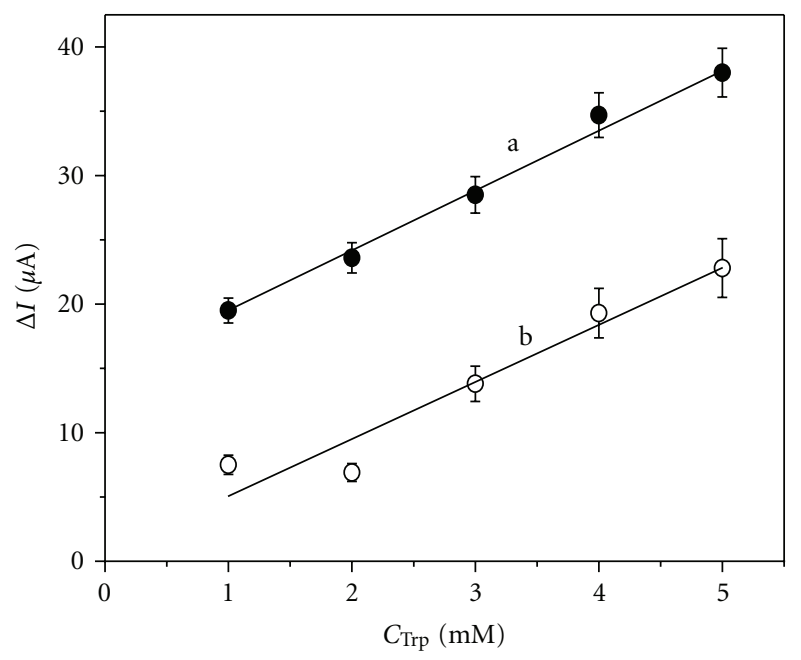

FIGURE 5: The relative change of oxidation current peak for different concentration Trp enantiomers adsorbed on modified electrode (a) L-Trp and (b) D-Trp.

3.4. Application of the Enantioselective Electrode. A series of solutions were prepared by mixing L- and D-Trp at different fixed volume ratios. The modified electrodes were used to determine the relative peak current change of different mixture solutions with $2 \mathrm{mM}$ and $5 \mathrm{mM}$. Figure 6 showed that the compositions of L- and D-Trp could be determined from the calibration curves which showed good linearity. The consequence implied that the adsorption quantity of Trp enantiomers on the interface gradually decreased with the increasing volume ratio of D-Trp. The results were consistent with prior measurements. 


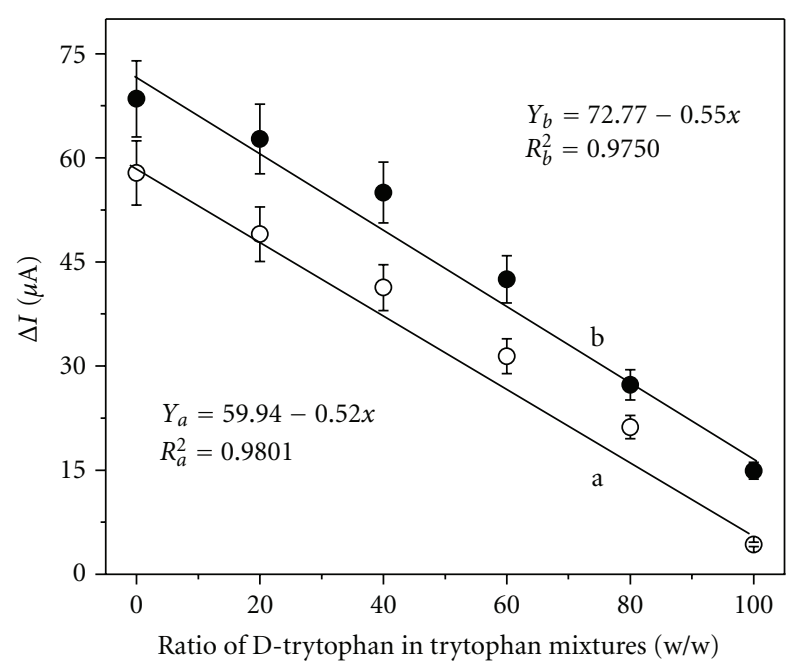

FIGURE 6: The relative change of the modified electrode oxidation current peak with enantiomeric composition of L- and D-Trp at different concentrations: (a) $2 \mathrm{mM}$ and (b) $5 \mathrm{mM}$.

\section{Conclusion}

The paper provided a simple scheme to discriminate $\operatorname{Trp}$ enantiomers via electrochemical method. The $(1 \mathrm{R}, 2 \mathrm{R})$ ADE chiral selective interface with simple preparation, fast detection and chiral recognition for L- and D-Trp was covalently attached on a self-assembled MPA monolayer. The stereoselectivity of the interface for L- and D-Trp was detected through the changes of current and resistance. All consequences showed that the modified electrode exhibited responses for Trp enantiomers, especially for L-Trp, and it had the potential to discriminate Trp enantiomers in pharmaceutical assays.

\section{Acknowledgment}

This work was supported by the National Natural Science Foundation of China (nos. 20732003 and 20972128).

\section{References}

[1] D. Berthier, T. Buffeteau, J. Léger, R. Oda, and I. Huc, "From chiral counterions to twisted membranes," Journal of the American Chemical Society, vol. 122, no. 45, pp. 13486-13494, 2002.

[2] J. A. Switzer, H. M. Kothari, P. H. Poizot, S. H. Nakanishi, and E. W. Bohannan, "Enantiospecific electrodeposition of a chiral catalyst," Nature, vol. 425, no. 6957, pp. 490-493, 2003.

[3] R. Q. Wang, T. T. Ong, and S. C. Ng, "Synthesis of cationic $\beta$-cyclodextrin derivatives and their applications as chiral stationary phases for high-performance liquid chromatography and supercritical fluid chromatography," Journal of Chromatography A, vol. 1203, no. 2, pp. 185-192, 2008.

[4] J. Ide, T. Nakamoto, and T. Moriizumi, "Discrimination of aromatic optical isomers using quartz-resonator sensors," Sensors and Actuators, vol. 49, no. 1-2, pp. 73-78, 1995.
[5] C. Xu, S. C. Ng, and H. S. O. Chan, "Self-assembly of perfunctionalized $\beta$-cyclodextrins on a quartz crystal microbalance lor real-time chiral recognition," Langmuir, vol. 24, no. 16, pp. 9118-9124, 2008.

[6] T. Nakanishi, N. Yamakawa, T. Asahi, N. Shibata, B. Ohtani, and T. Osaka, "Chiral discrimination between thalidomide enantiomers using a solid surface with two-dimensional chirality," Chirality, vol. 16, pp. S36-S39, 2004.

[7] H. Eun and Y. Umezawa, "Quartz crystal microbalance for $L^{-}$ leucine sensing based on growth of $L^{-}$-leucine crystals immobilized on a monolayer of 11-mercaptoundecanoic acid," Analytica Chimica Acta, vol. 413, no. 1-2, pp. 223-227, 2000.

[8] S. Hazarika, "Enantioselective permeation of racemic alcohol through polymeric membrane," Journal of Membrane Science, vol. 310, no. 1-2, pp. 174-183, 2008.

[9] Z. Wang, G. Luo, J. Chen, S. Xiao, and Y. Wang, "Carbon nanotubes as separation carrier in capillary electrophoresis," Electrophoresis, vol. 24, no. 24, pp. 4181-4188, 2003.

[10] S. Allenmark, "Induced circular dichroism by chiral molecular interaction," Chirality, vol. 15, no. 5, pp. 409-422, 2003.

[11] F. Gao, W. J. Ruan, J. M. Chen, Y. H. Zhang, and Z. A. Zhu, "Spectroscopy, NMR and DFT studies on molecular recognition of crown ether bridged chiral heterotrinuclear salen Zn(II) complex," Spectrochimica Acta A, vol. 62, no. 4-5, pp. 886-895, 2005.

[12] H. S. Guo, J. M. Kim, S. M. Chang, and W. S. Kim, "Chiral recognition of mandelic acid by l-phenylalanine-modified sensor using quartz crystal microbalance," Biosensors and Bioelectronics, vol. 24, no. 9, pp. 2931-2934, 2009.

[13] A. Q. Mi, Z. Y. Wang, X. M. Zhang, F. M. Fu, and Y. Z. Jiang, "Study on asymmetric reformatskii reaction," Acta Chimica Sinica, vol. 56, no. 7, pp. 719-728, 1998.

[14] A. Q. Mi, Z. Y. Wang, X. M. Zhang, and Y. Z. Jiang, "Asymmetric synthesis XXVI: catalytic enantioselective syntheses of $\beta$-hydroxy esters via double chiral induction in asymmetric reformatsky reactions," Synthetic Communications, vol. 27, no. 9, pp. 1469-1473, 1997.

[15] C. Q. Yin, B. J. He, S. R. Li, Y. Q. Liu, and Z. W. Bai, "Immobilization of (1S,2R)-(+)-2-amino-1,2-di-phenylethanol derivates on aminated silica gel with different linkages as chiral stationary phases and their enantioseparation evaluation by HPLC," Chirality, vol. 21, no. 4, pp. 442-448, 2009.

[16] A. R. Fiorucci and E. T. G. Cavalheiro, "The use of carbon paste electrode in the direct voltammetric determination of tryptophan in pharmaceutical formulations," Journal of Pharmaceutical and Biomedical Analysis, vol. 28, no. 5, pp. 909-915, 2002.

[17] Y. Suzuki, T. Suda, K. Furuhashi et al., "Increased serum kynurenine/tryptophan ratio correlates with disease progression in lung cancer," Lung Cancer, vol. 67, no. 3, pp. 361-365, 2010.

[18] A. J. Willem Van der Does, "The effects of tryptophan depletion on mood and psychiatric symptoms," Journal of Affective Disorders, vol. 64, no. 2-3, pp. 107-119, 2001.

[19] D. Wang, P. Wei, and P. Ouyang, "The research progress of Dtryptophan," Chemical Industry and Engineer Progress, vol. 21, pp. 103-105, 2002.

[20] B. B. Prasad, R. Madhuri, M. P. Tiwari, and P. S. Sharma, "Enantioselective recognition of D- and L-tryptophan by imprinted polymer-carbon composite fiber sensor," Talanta, vol. 81, no. 1-2, pp. 187-196, 2010.

[21] H. Li, F. Li, C. Han, Z. Cui, G. Xie, and A. Zhang, "Highly sensitive and selective tryptophan colorimetric sensor based on 4,4-bipyridine-functionalized silver nanoparticles," Sensors and Actuators B, vol. 145, no. 1, pp. 194-199, 2010. 
[22] D. Tang, R. Yuan, Y. Chai et al., "Preparation and application of a potentiometric platinum munosensor based on colloidal gold and polyvinyl butyralJ," Southwest China Normal University, vol. 29, pp. 843-847, 2004.

[23] X. Che, R. Yuan, Y. Chai, J. Li, Z. Song, and W. Li, "Amperometric glucose biosensor based on prussian blue-multiwall carbon nanotubes composite and hollow PtCo nanochains," Electrochimica Acta, vol. 55, no. 19, pp. 5420-5427, 2010.

[24] F. Scholz and R. Gulaboski, "Gibbs energies of transfer of chiral anions across the interface water-chiral organic solvent determined with the help of three-phase electrodes," Faraday Discussions, vol. 129, pp. 169-177, 2005.

[25] V. Mirčeski, R. Gulaboski, and F. Scholz, "Determination of the standard gibbs energies of transfer of cations across the nitrobenzenejwater interface utilizing the reduction of iodine in an immobilized nitrobenzene droplet," Electrochemistry Communications, vol. 4, pp. 814-819, 2002.

[26] V. Mirčeski, F. Quentel, and M. L. Her, "Chiral recognition based on the kinetics of ion transfers across liquid/liquid interface," Electrochemistry Communications, vol. 11, no. 6, pp. 1262-1264, 2009. 


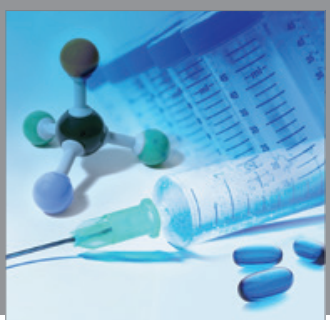

International Journal of

Medicinal Chemistry

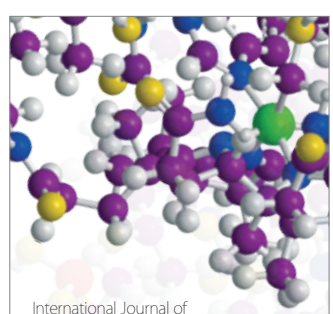

Carbohydrate Chemistry

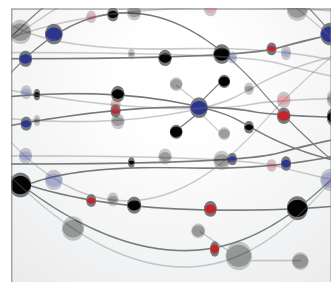

The Scientific World Journal
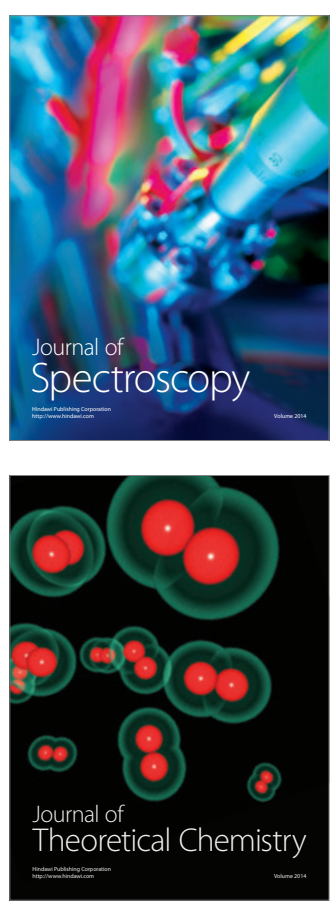
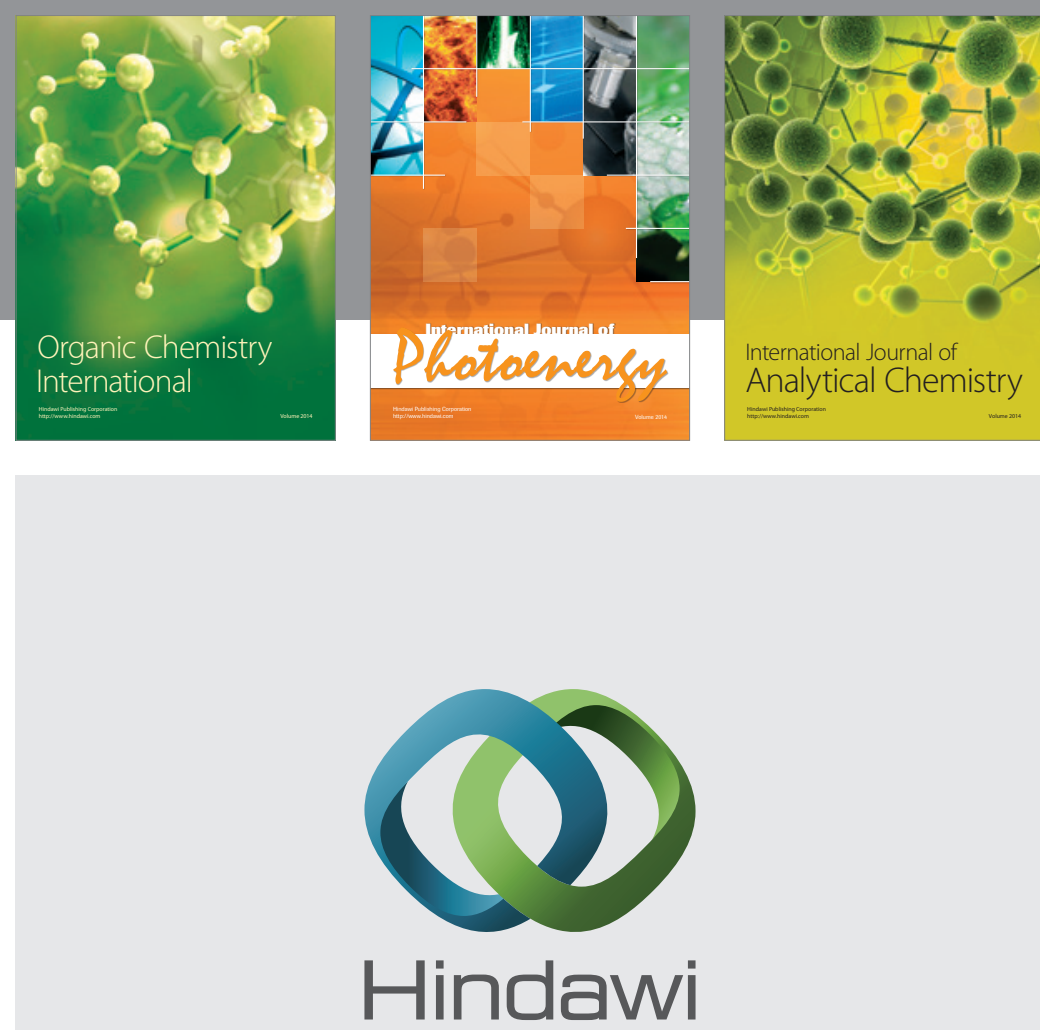

Submit your manuscripts at

http://www.hindawi.com
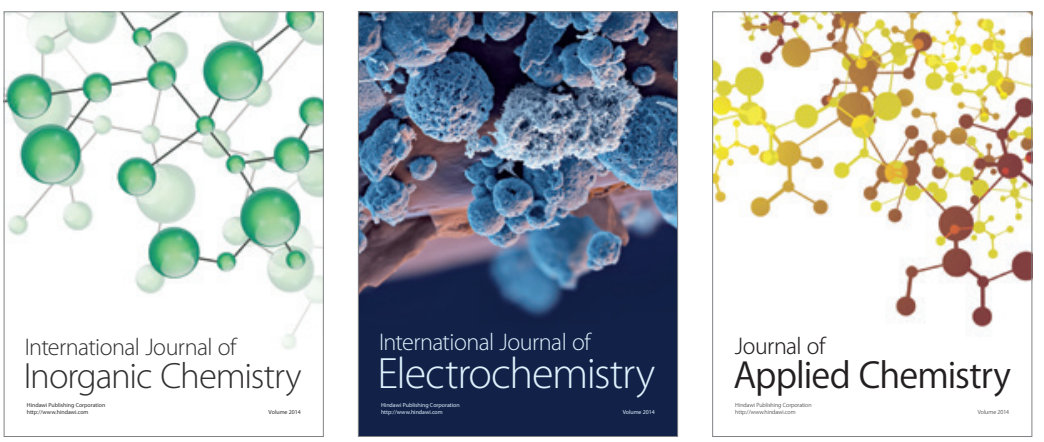

Journal of

Applied Chemistry
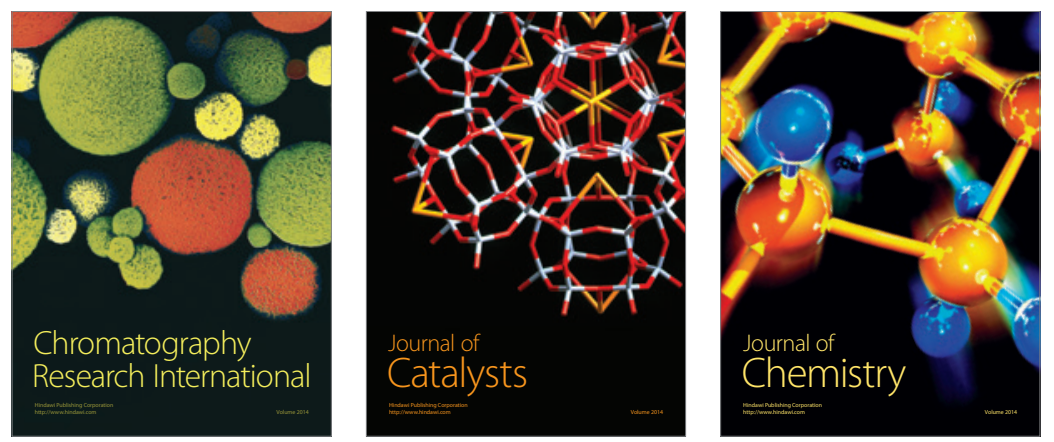
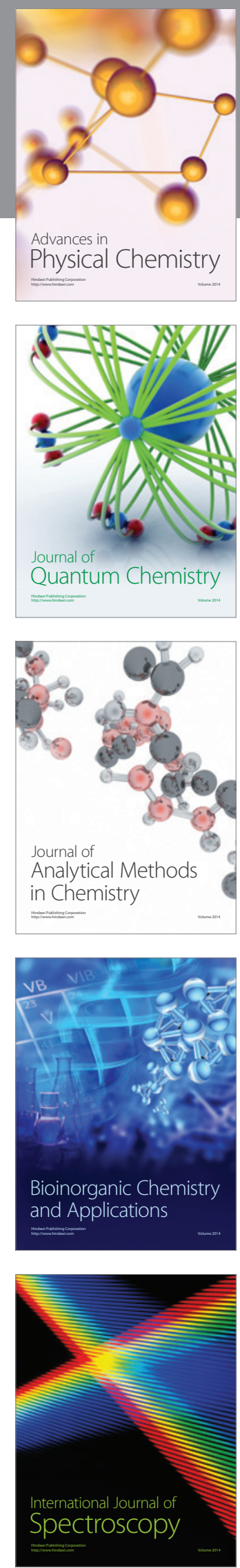